\title{
Breastfeeding is associated with waist-to- height ratio in young adults
}

\author{
Adam D. Bohr ${ }^{1 *}$, Jason D. Boardman², Benjamin W. Domingue ${ }^{3}$ and Matthew B. McQueen ${ }^{4}$
}

\begin{abstract}
Background: The current study investigated the association between breastfeeding and adult weight distribution using an emerging indicator of weight distribution, the waist-to-height ratio (WHtR).

Methods: The study sample consisted of two subsamples of individuals that were part of the National Longitudinal Study of Adolescent Health. One sample $(n=1$ 179) consisted of individuals from the sibling pair data. A second sample ( $n=4$ 648) consisted of individuals that were not part of the paired data. Regression models were constructed to establish if there was a relationship between breastfeeding and two measures of weight distribution: WHtR and body mass index (BMI). Controls for parental socioeconomic status, maternal smoking, race, sex, age, birth weight, maternal BMI, genetic ancestry, and a genetic risk score (GRS) for obesity were included. In addition, a behavioral risk score (BRS) was constructed to control for other residual confounding factors.

Results: A significant, inverse relationship between breastfeeding and adult WHtR persisted in models constructed from the sibling pair sample $(P=0.002)$ and unrelated sample $(P<0.0001)$. This association remained significant with the inclusion of ancestry principal components, GRS, and a measure of maternal obesity.

Conclusions: The moderate association between breastfeeding and weight distribution persists into adulthood while controlling for potential confounders. This paper also provides evidence that the WHtR may be a superior outcome measure to BMI in studies investigating breastfeeding and obesity.
\end{abstract}

Keywords: Breastfeeding, Obesity, Residual Confounding, Waist-to-Height Ratio

\section{Background}

The prevalence of obesity and overweight has increased significantly over the past two decades. Among nonHispanic white men, the prevalence of obesity increased from $20.3 \%$ in the years $1994-1998$ to $35.7 \%$ by $2009-$ 2010 [1]. Recent estimates place the number of overweight at one billion worldwide, of which 300 million are classified as obese [2]. As such, the obesity epidemic has become one of the most pressing public health issues facing global populations.

Increases in obesity prevalence have been linked to an increasingly sedentary lifestyle and consumption of a low quality diet, but these factors alone do not completely explain the nature of the epidemic, nor the fact that some are more prone to becoming obese and retaining weight than others $[3,4]$. One of the most

\footnotetext{
* Correspondence: Adam.Bohr@Colorado.edu

${ }^{1}$ University of Colorado Boulder, 4185 47th St., Unit C, Boulder, CO 80301, USA

Full list of author information is available at the end of the article
}

important early developmental factors of interest is breastfeeding. A recent study from a sample of overweight adolescents revealed that breastfeeding of an infant is associated with lower incidence of obesity and complications related to metabolic syndrome in the offspring [5]. Other studies have revealed that longer duration of breastfeeding of an infant may be protective against obesity in childhood or reduce the risk of being overweight in childhood [6-9]. Researchers have identified several physiological links between breastfeeding and body size of the offspring. In addition to fatty acids, vitamins, and minerals, breast milk also contains a diverse population of bacteria that colonize the intestinal tract of the infant and may have protective benefits against weight gain throughout life [10]. Breastfeeding duration may also delay the introduction of solid foods for the infant, which has been linked to childhood obesity in some cohorts [11]. However, other studies have found this not to be predictive of obesity [10]. 
Despite these findings, studies that link breastfeeding to weight status often come under scrutiny. One reason is that the evidence comes solely from observational studies, as randomized trials for breastfeeding would be unethical [12]. The observational studies that do exist have potentially serious problems with selection bias (e.g., those that are most likely to breastfeed may also be the most likely to lead a lifestyle that is related to a healthy body mass index (BMI)) and residual confounding, as breastfeeding is strongly associated with important characteristics of mothers. [6, 12-14] Nevertheless, recent studies have attempted to reduce residual confounding and selection bias by using sibling pairs that were discordant for breastfeeding $[15,16]$. They found that the relationship between breastfeeding and BMI observed in between-families analyses was no longer significant in the within-family analysis.

This study builds upon earlier work by using an emerging indicator of weight distribution, the waist-to-height ratio (WHtR), as the primary outcome variable to test this association [17, 18]. There is evidence that the WHtR may be a more accurate diagnostic tool for obesity-related chronic diseases than the more traditional BMI because it more accurately characterizes adiposity [18]. It is known that visceral fat is more metabolic and inflammatory than adipose tissue in other subcutaneous regions $[19,20]$. This concept is also supported by a meta-analysis performed by Ashwell et al. (2012) which showed that the WHtR consistently discriminated better for co-morbidities associated with obesity than BMI and waist circumference [17]. Further, in the context of early developmental factors, using height in combination with waist circumference may result in a more sensitive measure to detect early developmental factors associated with obesity. This is because poor fetal nutrition is associated with both short stature and overweight status [18]. We also perform analyses using BMI to allow for comparisons between previously conducted studies of breastfeeding and measures of body mass. Finally, on a subset of the overall sample, we utilize genome-wide association data to incorporate a genetic risk score (GRS) for BMI as well as measures of genetic ancestry.

\section{Methods}

\section{Participants}

The study utilized a sample of individuals from the National Longitudinal Study of Adolescent Health (Add Health) [21]. Add Health is a longitudinal study that investigates how social and environmental factors may influence health. It has followed a cohort of individuals through four waves of interviewing and testing since its inception during the 1994-1995 school year. The full Add Health study consisted of 20792 participants.
However, a substantial amount of this sample was not part of the Wave 1 parent interviews or measured for waist circumference and height at Wave 4 . In addition, genome-wide association data is available on the sibling pair (SP) sample only. Therefore, we analyzed a subsample of 1179 respondents with genome-wide data from the SP sample as well as 4648 respondents from the unrelated (UN) sample. Also, to ensure that the relatedness of the siblings was not impacting the associations observed in the SP sample, we randomly selected one participant from each sibling pair resulting in a third sample of 856 respondents and performed analyses on this sample. Variables from all four waves of interviews and measurements from the Add Health Study were used. In an effort to retain the largest possible sample size, missingness was allowed for two covariates in the analysis: birth weight and parental smoking.

\section{Data}

The specific Add Health variable code names used in this study are listed in the supplementary information section to this paper (Additional file 2: Table S1). The primary variables of interest in this study were breastfeeding, WHtR, and BMI. Breastfeeding data were extracted from the in-home parent interviews conducted during Wave 1 (baseline). The options provided for the breastfeeding question were "Never Breastfed", "0-3 Months", "3-6 Months", "6-9 Months", "9-12 Months", "12-24 Months", and "Greater than 24 Months." We removed any cases of missingness for breastfeeding from our analyses. In an effort to reduce any potential recall bias that may exist in the reporting of duration of breastfeeding, we opted to analyze breastfeeding only as a dichotomous variable. Individuals that were never breastfed were coded as " 0 " while those that were breastfed were coded as " 1 ".

Height $(\mathrm{cm})$ and waist circumference $(\mathrm{cm})$ were extracted from the Add Health Wave 4 anthropometric measurements. Waist circumference was measured to the nearest $0.5 \mathrm{~cm}$ at the superior border of the iliac crest. [21] The WHtR metric was calculated by dividing waist circumference by height. BMI values were also taken from Wave 4 data. As with breastfeeding, we removed any cases from our analyses that were missing for WHtR and BMI.

To control for behavioral differences between individuals, we utilized risk scores constructed from a variety of indicators. The risk scores were calculated using the $\mathrm{R}$ package, pcaMethods, and allowed for missing data [22]. For this study, the behavioral risk scores (BRS) are the first three principal components derived from a cluster of items regarding diet, sleep, and exercise. The BRS components were validated using both Wave $4 \mathrm{WHtR}$ and BMI. The specific Add 
Health variables used and details on how the risk scores were constructed can be found in the supplementary information (Additional file 1 and Additional file 2: Table S2).

A genetic risk score (GRS) was calculated for each individual in the SP data set. Genome-wide association studies have identified 31 single nucleotide polymorphisms (SNPs) in individuals of European descent and 8 SNPs in individuals of African American descent that are associated with BMI [23, 24]. These SNPs were used to compute risks scores, and analyses were conducted testing the association of these risk scores with BMI, obesity, WHtR, and change in BMI over time. A more in depth description of these risk scores and their construction using the SP sample can be found in Domingue et al. [25]. To develop our measure of genetic ancestry, we used KING to identify clusters of individuals based upon genetic similarity [26]. KING uses multidimensional scaling (MDS) with Euclidean distance to generate principal coordinates (PCs) that can be used to identify population substructure. A full description of the derivation of the ancestry PCs can be found in McQueen et al. [27].

In addition to the calculated risk scores, we used the following controls: birth weight (lb.); parental SES; parental smoking; maternal obesity; gender; age; and race. Birth weights for the subjects were obtained from the Wave 1 in-home parent interviews. Because low birth weight is hypothesized to be associated with higher WHtR in adulthood, birth weight was assessed as a categorical variable that compared low birth weight subjects to normal birth weight subjects. Low birth weight status was assigned to subjects that weighed less than 2 $500 \mathrm{~g}(5.5 \mathrm{lb}$.). As stated above, this variable allowed for missingness and was analyzed as a factor, with low birth weight and missing birth weight compared to the referent category, normal birth weight.

Self-reported parental smoking status was also extracted from the Wave 1 in-home parent interviews and analyzed as a factor, allowing for missingness, with "yes" for smoking and missingness for smoking compared to the referent group of nonsmoking. For a measure of maternal obesity, we used the question "Is the biological mother obese?" from the Wave 1 in-home parent interview. This variable was dichotomous, and a response of "no" was coded as "0" while "yes" was coded as "1". Selfreported race was analyzed as a factor, comparing Hispanic, non-Hispanic white, and non-Hispanic Asian/Native American to the referent group non-Hispanic African American. Parental SES was assessed with selfreported household income at Wave 1 of the study and was analyzed as a dichotomous variable. We coded those that reported income less than the median income from the overall sample (\$38 000) as low SES ("0") and those that reported income of greater than the median as high SES ("1").

Descriptive statistics in total and stratified by breastfeeding status for sex, race, parental smoking, low birth weight occurrence, parental SES, maternal obesity, BMI, and WHtR for the two samples can be found in Tables 1 and 2. The average age of the participants at Wave 4 was 28.49 years with a range of 24.32-33.59 years. Breastfeeding initiation was $49.2 \%$ in our UN sample and $39.4 \%$ in our SP sample. In reviewing the descriptive statistics for this study, it is worth commenting on certain elements of the sample. Rates of breastfeeding initiation were $26.5 \%$ in 1970 and rose to $61.9 \%$ in 1982 [28]. As the participants in the Add Health study were born between 1976 and 1984, it is reasonable that the initiation rates in our samples would be between these two rates. In addition, there are some racial disparities in the initiation of breastfeeding. African Americans comprise $18.4 \%$ of the individuals in the UN sample but only $11.68 \%$ of the individuals that were breastfed. This disparity was observed in the SP sample as well. This is consistent with previous literature that has demonstrated a lower rate of breastfeeding initiation in African Americans, while there are not large discrepancies between non-Hispanic white or Hispanics [29]. In addition, it may explain some of the difference in breastfeeding rates between our two samples, as the SP sample was $34.18 \%$ African American and the UN sample was $18.42 \%$ African American. Finally, there were differences in the rates of low birth weight between the two samples (UN Sample: 4.65 \%; SP Sample: 13.57 \%) that are likely attributed to the oversampling of African Americans.

\section{Statistical analysis \\ Unrelated sample (UN)}

OLS regression models were used to investigate the relationship between breastfeeding and measures of weight distribution. For both WHtR and BMI, breastfeeding was the primary predictor variable of interest. As noted above, breastfeeding was analyzed as a dichotomous variable (ever breastfed coded as "1", never breastfed coded as " 0 ") in these models. Models constructed from the UN data included all covariates with the exception of the genetic risk scores and ancestry principal components. We also tested for a maternal obesity and breastfeeding interaction to assess whether or not the association between breastfeeding and weight distribution was dependent on the weight status of the mother. This interaction term was not significant in any of our analyses and therefore excluded from the final models. Additionally, we tested for an interaction between breastfeeding and the genetic risk score that was also not significant and excluded from the final models. 
Table 1 Descriptive statistics for participants from the add health study, 1994-2008. Unrelated sample

\begin{tabular}{|c|c|c|c|c|c|c|}
\hline & & & Breastfe & tus (Yes/N & & \\
\hline & Full sam & & No & & Yes & \\
\hline & 4648 & & 2362 & & 2286 & \\
\hline & n & $\%$ & $\mathrm{n}$ & $\%$ & $\mathrm{n}$ & $\%$ \\
\hline Males & 2116 & $45.52 \%$ & 1056 & $44.71 \%$ & 1060 & $46.37 \%$ \\
\hline Females & 2532 & $54.48 \%$ & 1306 & $55.29 \%$ & 1226 & $53.63 \%$ \\
\hline Race & & & & & & \\
\hline Non-Hispanic White & 2865 & $61.64 \%$ & 1368 & $57.92 \%$ & 1497 & $65.49 \%$ \\
\hline Non-Hispanic African American & 856 & $18.42 \%$ & 589 & $24.94 \%$ & 267 & $11.68 \%$ \\
\hline Non-Hispanic Asian/Native American & 259 & $5.57 \%$ & 89 & $3.77 \%$ & 170 & $7.44 \%$ \\
\hline Hispanic & 666 & $14.33 \%$ & 315 & $13.34 \%$ & 351 & $15.35 \%$ \\
\hline Parent smoker & & & & & & \\
\hline No & 714 & $15.36 \%$ & 370 & $15.66 \%$ & 344 & $15.05 \%$ \\
\hline Yes & 1328 & $28.57 \%$ & 838 & $35.48 \%$ & 490 & $21.43 \%$ \\
\hline Missing & 2606 & $56.07 \%$ & 1154 & $48.86 \%$ & 1452 & $63.52 \%$ \\
\hline Low birth weight & & & & & & \\
\hline No & 4351 & $93.61 \%$ & 2155 & $91.24 \%$ & 2196 & $96.06 \%$ \\
\hline Yes & 216 & $4.65 \%$ & 146 & $6.18 \%$ & 70 & $3.06 \%$ \\
\hline Missing & 81 & $1.74 \%$ & 61 & $2.58 \%$ & 20 & $0.87 \%$ \\
\hline Parent socioeconomic status & & & & & & \\
\hline Below median income & 2101 & $45.20 \%$ & 1250 & $52.92 \%$ & 851 & $37.23 \%$ \\
\hline Above median income & 2547 & $54.80 \%$ & 1112 & $47.08 \%$ & 1435 & $62.77 \%$ \\
\hline Maternal obesity & & & & & & \\
\hline No & 3790 & $81.54 \%$ & 1901 & $80.48 \%$ & 1889 & $82.63 \%$ \\
\hline Yes & 858 & $18.46 \%$ & 461 & $19.52 \%$ & 397 & $17.37 \%$ \\
\hline & Mean & SE & Mean & SE & Mean & SE \\
\hline Body mass index & 29.007 & 0.112 & 29.803 & 0.165 & 28.185 & 0.148 \\
\hline Waist-to-height ratio & 0.575 & 0.002 & 0.589 & 0.002 & 0.562 & 0.002 \\
\hline
\end{tabular}

Finally, we used the Add Health Wave 1 sample weights for all models in the UN sample. The statistical power for these models was as follows: WHtR Outcome, (1 $\beta)<0.001$; BMI Outcome $(1-\beta)<0.001$.

In addition to the linear models, we also constructed logistic regression models to further investigate the decrease in risk of obesity associated with breastfeeding in both samples. For this analysis, we created a dichotomous variable to indicate obesity status $(\mathrm{BMI} \geq 30)$. All analyses and calculations were done using $\mathrm{R}$ version 2.15.3 via the RStudio platform, version 0.97.320 [30].

\section{Sibling Pair Sample (SP)}

OLS regression models were also constructed from the SP sample. These models included all of the covariates that were tested in the UN sample and tested the outcomes WHtR, BMI, and BMI $\geq 30$. In addition, these models included the PCs for genetic ancestry and the
GRS as controls. The statistical power for these models was as follows: WHtR Outcome, $(1-\beta)=0.018$; BMI Outcome $(1-\beta)=0.177$.

\section{Subset of Sibling Pair Sample (SSP)}

Finally, OLS regression models were also constructed from a subset of the SP sample. One participant was randomly selected from each of the sibling pairs, and the OLS models described for the SP sample were also constructed from this subset. The statistical power for these models was as follows: WHtR Outcome, $(1-\beta)=0.093$; BMI Outcome $(1-\beta)=0.422$.

\section{Results}

Primary outcomes and exposures

The results of the multiple linear regression models from the UN and SP samples are presented in Tables 3 and 4. In our UN sample, breastfeeding was associated 
Table 2 Descriptive statistics for participants from the Add Health Study, 1994-2008. Sibling pair sample

\begin{tabular}{|c|c|c|c|c|c|c|}
\hline & & & Breastfe & tus (Yes/N & & \\
\hline & Full san & & No & & Yes & \\
\hline & 1179 & & 715 & & 464 & \\
\hline & $\mathrm{n}$ & $\%$ & $\mathrm{n}$ & $\%$ & $\mathrm{n}$ & $\%$ \\
\hline Males & 581 & $49.28 \%$ & 350 & $48.95 \%$ & 231 & $49.78 \%$ \\
\hline Females & 598 & $50.72 \%$ & 365 & $51.05 \%$ & 233 & $50.22 \%$ \\
\hline Race & & & & & & \\
\hline Non-Hispanic White & 625 & $53.01 \%$ & 321 & $44.90 \%$ & 304 & $65.52 \%$ \\
\hline Non-Hispanic African American & 403 & $34.18 \%$ & 333 & $46.57 \%$ & 70 & $15.09 \%$ \\
\hline Non-Hispanic Asian/Native American & 35 & $2.97 \%$ & 8 & $1.12 \%$ & 27 & $5.82 \%$ \\
\hline Hispanic & 116 & $9.84 \%$ & 53 & $7.41 \%$ & 63 & $13.58 \%$ \\
\hline Parent smoker & & & & & & \\
\hline No & 194 & $16.45 \%$ & 116 & $16.22 \%$ & 78 & $16.81 \%$ \\
\hline Yes & 369 & $31.30 \%$ & 270 & $37.76 \%$ & 99 & $21.34 \%$ \\
\hline Missing & 616 & $52.25 \%$ & 329 & $46.01 \%$ & 287 & $61.85 \%$ \\
\hline Low birth weight & & & & & & \\
\hline No & 984 & $83.46 \%$ & 558 & $78.04 \%$ & 426 & $91.81 \%$ \\
\hline Yes & 160 & $13.57 \%$ & 126 & $17.62 \%$ & 34 & $7.33 \%$ \\
\hline Missing & 35 & $2.97 \%$ & 31 & $4.34 \%$ & 4 & $0.86 \%$ \\
\hline Parent socioeconomic status & & & & & & \\
\hline Below median income & 661 & $56.06 \%$ & 453 & $63.36 \%$ & 208 & $44.83 \%$ \\
\hline Above median income & 518 & $43.94 \%$ & 262 & $36.64 \%$ & 256 & $55.17 \%$ \\
\hline Maternal obesity & & & & & & \\
\hline No & 909 & $77.10 \%$ & 560 & $78.32 \%$ & 349 & $75.22 \%$ \\
\hline Yes & 270 & $22.90 \%$ & 155 & $21.68 \%$ & 115 & $24.78 \%$ \\
\hline & Mean & SE & Mean & SE & Mean & SE \\
\hline Body mass index & 29.321 & 0.223 & 29.880 & 0.300 & 28.459 & 0.325 \\
\hline Waist-to-height ratio & 0.580 & 0.003 & 0.588 & 0.004 & 0.566 & 0.004 \\
\hline
\end{tabular}

with a decrease in both WHtR and BMI. All other factors being equal, breastfeeding was associated with a decrease in predicted adult WHtR of 0.012 and in predicted adult BMI of 0.548 . We observed a consistent effect in the SP sample, as breastfeeding was associated with a decrease in both WHtR and BMI. Once again, all other factors being equal, breastfeeding was associated with a decrease in predicted adult WHtR of 0.020 and predicted adult BMI of 1.049. As anticipated, the GRS is a highly significant predictor of body weight measures in all models constructed from the SP sample. The third principal component for ancestry was a significant predictor of both WHtR and BMI in the SP sample while the fifth principal component was predictive of BMI only. All three principal components of the BRS were significant predictors of body weight measures in our UN sample, but only the first principal component remained significant with the inclusion of the GRS in the SP sample.
The results from the logistic regression model are presented in Table 5 and include calculated odds ratios, $95 \%$ confidence intervals, and $P$ values. Consistent with the results of the linear models, breastfeeding was associated with a decreased risk of obesity in the UN sample $(\mathrm{OR}=0.854,95 \% \mathrm{CI}=(0.75,0.97))$. With all other factors being equal, having been breastfed was associated with a 0.854 odds of obesity compared to those that were not breastfed. Conversely, those that were not breastfed were 1.171 times more likely to be obese than those that were breastfed with all else being equal. Breastfeeding was associated with the same odds ratio in the SP sample, though it was not statistically significant $(\mathrm{OR}=0.854,95 \% \mathrm{CI}=(0.64,1.13))$.

The results of the multiple linear regression models and logistic regression model constructed from the subset of the SP sample are presented in Table 6. Consistent with the results from both the UN sample and full SP sample, breastfeeding was associated with a decrease in 
Table 3 Regression results for models constructed from unrelated sample, $n=4648$, Add Health Study 1994-2008

\begin{tabular}{|c|c|c|c|c|c|c|}
\hline & \multicolumn{3}{|c|}{ Waist-to-height ratio outcome } & \multicolumn{3}{|c|}{ Body mass index outcome ${ }^{* *}$} \\
\hline & $\mathrm{b}$ & SE & $P$ value & $\mathrm{b}$ & SE & $P$ value \\
\hline Intercept & 0.541 & 0.027 & $<0.0001$ & 29.085 & 2.082 & $<0.0001$ \\
\hline Sex & 0.028 & 0.003 & $<0.0001$ & -0.061 & 0.220 & 0.783 \\
\hline Age & 0.001 & 0.001 & 0.516 & 0.075 & 0.069 & 0.278 \\
\hline Breastfeeding & -0.012 & 0.003 & $<0.0001$ & -0.516 & 0.221 & 0.020 \\
\hline Birthweight $>2500 \mathrm{~g}$ & Reference & Reference & Reference & Reference & Reference & Reference \\
\hline Birthweight $<2500 \mathrm{~g}$ & 0.001 & 0.006 & 0.926 & -0.965 & 0.485 & 0.047 \\
\hline Birthweight missing & 0.009 & 0.011 & 0.427 & -0.298 & 0.829 & 0.719 \\
\hline Parent nonsmoker & Reference & Reference & Reference & Reference & Reference & Reference \\
\hline Parent smoker & 0.010 & 0.004 & 0.024 & 0.808 & 0.327 & 0.014 \\
\hline Parent smoking missing & -0.011 & 0.004 & 0.005 & -0.769 & 0.303 & 0.011 \\
\hline Non-Hispanic African American & Reference & Reference & Reference & Reference & Reference & Reference \\
\hline Non-Hispanic White & -0.013 & 0.004 & 0.003 & -1.911 & 0.330 & $<0.0001$ \\
\hline Non-Hispanic Asian/Native American & -0.014 & 0.009 & 0.115 & -2.185 & 0.671 & 0.001 \\
\hline Hispanic & 0.010 & 0.006 & 0.081 & -0.292 & 0.430 & 0.498 \\
\hline Behavioral risk score 4_1 & -0.015 & 0.002 & $<0.0001$ & -0.773 & 0.125 & $<0.0001$ \\
\hline Behavioral risk score 4_2 & -0.007 & 0.003 & 0.007 & -0.399 & 0.192 & 0.038 \\
\hline Behavioral risk score 4_3 & -0.008 & 0.003 & 0.003 & -0.377 & 0.197 & 0.055 \\
\hline Parent socioeconomic status & -0.002 & 0.001 & $<0.0001$ & -0.141 & 0.043 & 0.001 \\
\hline Maternal obesity & 0.055 & 0.004 & $<0.0001$ & 4.525 & 0.271 & $<0.0001$ \\
\hline
\end{tabular}

b (Coefficient) SE (Standard Error)

* R-Squared $=0.13$

** R-Squared $=0.10$

both WHtR and BMI. All other factors being equal, breastfeeding was associated with a decrease in predicted adult WHtR of 0.025 and in predicted adult BMI of 1.604. In addition, the results of the logistic regression predicting $\mathrm{BMI} \geq 30$ showed a significant association with breastfeeding $(\mathrm{OR}=0.695,95 \% \mathrm{CI}=(0.50,0.97))$. With all other factors being equal, those that were not breastfed were 1.43 times more likely to be obese than those that were breastfed.

\section{Other covariates}

Low birth weight was associated with decreased BMI in our UN sample but was not a significant predictor in any other models. Parental smoking was a significant predictor of both BMI, WHtR, and $\mathrm{BMI} \geq 30$ in the UN sample, but was not significant in the SP sample. Several of our racial categories were significant predictors of BMI in the UN sample. Reporting non-Hispanic white or non-Hispanic Asian/Native American was associated with a decrease in predicted BMI when compared with the referent group, non-Hispanic African American. However, this effect was only seen for those reporting white for the WHtR outcome. Of particular note, race was not a significant predictor in any of models from the SP sample or the subset from the SP sample. Our measure of parental SES was inversely related to WHtR and BMI in the UN sample and was trending in the SP sample. Parental SES was also a significant predictor of WHtR and was trending for BMI in the subset of the SP sample. Our variable for maternal obesity was a strong positive predictor of $\mathrm{BMI}, \mathrm{WHtR}$, and $\mathrm{BMI} \geq 30$ in all samples.

\section{Discussion}

Previous research has linked a variety of early childhood factors to the health status of adults [31]. A great deal of recent interest has focused on breastfeeding as an important determinant of health status into adulthood, and our study contributes to this body of work in several important ways. Few studies investigating the role breastfeeding use the WHtR as an outcome as opposed to BMI, and to our knowledge none have done so with adults. This is important because, while there are debates about the value of the BMI in epidemiologic research, the WHtR has been shown to be a superior indicator of physical composition as it describes the distribution of weight. [17-20] As such the inclusion of this measure enhances our understanding of the role of breastfeeding for the health of populations. 
Table 4 Regression results for models constructed from sibling pair sample, $n=1179$, Add Health Study 1994-2008

\begin{tabular}{|c|c|c|c|c|c|c|}
\hline & Waist-to-he & tio outcome & & Body mass & butcome $e^{* *}$ & \\
\hline & $\mathrm{b}$ & SE & $P$ value & $\mathrm{b}$ & SE & $P$ value \\
\hline Intercept & 0.392 & 0.063 & $<0.0001$ & 20.161 & 4.640 & $<0.0001$ \\
\hline Sex & 0.037 & 0.006 & $<0.0001$ & 0.301 & 0.477 & 0.528 \\
\hline Age & 0.000 & 0.002 & 0.838 & -0.063 & 0.132 & 0.634 \\
\hline Breastfeeding & -0.020 & 0.006 & 0.002 & -1.049 & 0.473 & 0.027 \\
\hline Birthweight >2500 g & Reference & Reference & Reference & Reference & Reference & Reference \\
\hline Birthweight <2500 g & 0.001 & 0.008 & 0.927 & -0.186 & 0.629 & 0.768 \\
\hline Birthweight Missing & 0.006 & 0.017 & 0.726 & 0.384 & 1.261 & 0.761 \\
\hline Parent nonsmoker & Reference & Reference & Reference & Reference & Reference & Reference \\
\hline Parent smoker & 0.008 & 0.009 & 0.334 & 0.322 & 0.648 & 0.619 \\
\hline Parent smoking missing & 0.007 & 0.008 & 0.375 & 0.686 & 0.598 & 0.251 \\
\hline Non-Hispanic African American & Reference & Reference & Reference & Reference & Reference & Reference \\
\hline Non-Hispanic White & -0.014 & 0.027 & 0.604 & -1.634 & 2.004 & 0.415 \\
\hline Non-Hispanic Asian/Native American & 0.025 & 0.047 & 0.589 & 3.625 & 3.452 & 0.294 \\
\hline Hispanic & -0.023 & 0.026 & 0.382 & -1.695 & 1.918 & 0.377 \\
\hline Behavioral risk score 4_1 & 0.016 & 0.004 & $<0.0001$ & 0.757 & 0.265 & 0.004 \\
\hline Behavioral risk score 4_2 & -0.001 & 0.004 & 0.787 & -0.206 & 0.282 & 0.466 \\
\hline Behavioral risk score 4_3 & -0.002 & 0.004 & 0.564 & 0.123 & 0.303 & 0.685 \\
\hline Parent socioeconomic status & -0.010 & 0.006 & 0.100 & -0.839 & 0.462 & 0.070 \\
\hline Maternal obesity & 0.047 & 0.007 & $<0.0001$ & 3.932 & 0.508 & $<0.0001$ \\
\hline Genetic risk score & 0.005 & 0.001 & $<0.0001$ & 0.402 & 0.065 & $<0.0001$ \\
\hline Ancestry principal component 1 & 0.273 & 0.544 & 0.617 & 10.394 & 40.325 & 0.797 \\
\hline Ancestry principal component 2 & -0.392 & 0.348 & 0.261 & -4.349 & 25.792 & 0.866 \\
\hline Ancestry principal component 3 & 0.769 & 0.215 & $<0.001$ & 57.713 & 15.944 & $<0.001$ \\
\hline Ancestry principal component 4 & -0.034 & 0.150 & 0.822 & 0.989 & 11.076 & 0.929 \\
\hline Ancestry principal component 5 & 0.366 & 0.187 & 0.0505 & 32.468 & 13.836 & 0.0191 \\
\hline
\end{tabular}

b (Coefficient) SE (Standard Error)

${ }^{*}$ R-Squared $=0.18$

** R-Squared $=0.14$

Second, the Add Health study has several features that enhance the validity of our findings. For example, BMI and WHtR were both measured in the field by trained technicians rather than self-reported. The design of the Add Health study allows for opportunity to make true population inferences not only about prevalence and incidence but more importantly about key associational parameters such as breastfeeding and adult health. The richness of these data allow us to include detailed and comprehensive measures of behavioral and environmental risks, such as SES, diet, and exercise, that may confound the association. In addition, our regression models had controls in place for race, parental SES, parental smoking, maternal obesity, and low birth weight. Finally, controlling for maternal obesity provides additional support that the association between breastfeeding and weight distribution is not dependent on family factors.
Finally, we were able to establish a consistent effect of breastfeeding on both WHtR and BMI in both Add Health subsamples. This finding was robust in the SP data set that included genetic risk factors for BMI/obesity as well as measures of genetic ancestry. We further tested this finding on a subset of the SP sample, demonstrating that this association was not due to the relatedness of the subjects. To our knowledge, this is the first study to assess the role of breastfeeding on weight distribution using measured genetic factors that could influence the risk of obesity. The robustness of the association between breastfeeding and measures of weight status controlling for genetics is novel and provides additional support for the association between breastfeeding and weight distribution that has been reported elsewhere.

There are many characteristics of breastfeeding that may help to explain how feeding practice early in life 
Table 5 Results of logistic regression predicting BMI $\geq 30$ : Unrelated and sibling pair sample, Add Health Study, 1994-2008

\begin{tabular}{|c|c|c|c|c|c|c|}
\hline & \multicolumn{3}{|c|}{ Unrelated sample } & \multicolumn{3}{|c|}{ Sibling pair sample* } \\
\hline & $\mathrm{OR}$ & $95 \% \mathrm{Cl}$ & $P$ value & OR & $95 \% \mathrm{Cl}$ & $P$ value \\
\hline Sex & 0.991 & $(0.87,1.13)$ & 0.897 & 1.114 & $(0.84,1.48)$ & 0.451 \\
\hline Age & 1.032 & $(0.99,1.08)$ & 0.140 & 1.021 & $(0.94,1.10)$ & 0.613 \\
\hline Breastfeeding & 0.854 & $(0.75,0.97)$ & 0.018 & 0.854 & $(0.64,1.13)$ & 0.277 \\
\hline Birthweight >2500 g & Reference & Reference & Reference & Reference & Reference & Reference \\
\hline Birthweight <2500 g & 1.073 & $(0.80,1.44)$ & 0.635 & 1.162 & $(0.81,1.67)$ & 0.419 \\
\hline Birthweight missing & 0.614 & $(0.37,1.01)$ & 0.055 & 0.908 & $(0.43,1.93)$ & 0.801 \\
\hline Parent nonsmoker & Reference & Reference & Reference & Reference & Reference & Reference \\
\hline Parent smoker & 1.259 & $(1.04,1.53)$ & 0.021 & 0.909 & $(0.62,1.33)$ & 0.623 \\
\hline Parent smoking missing & 0.863 & $(0.72,1.03)$ & 0.106 & 0.874 & $(0.61,1.25)$ & 0.456 \\
\hline Non-Hispanic African American & Reference & Reference & Reference & Reference & Reference & Reference \\
\hline Non-Hispanic White & 0.665 & $(0.56,0.79)$ & $<0.0001$ & 0.439 & $(0.14,1.41)$ & 0.168 \\
\hline Non-Hispanic Asian/Native American & 0.528 & $(0.38,0.73)$ & $<0.0001$ & 0.682 & $(0.08,5.83)$ & 0.727 \\
\hline Hispanic & 1.055 & $(0.85,1.31)$ & 0.621 & 0.738 & $(0.24,2.27)$ & 0.596 \\
\hline Behavioral risk score 4_1 & 0.794 & $(0.73,0.86)$ & $<0.0001$ & 1.244 & $(1.06,1.47)$ & 0.009 \\
\hline Behavioral risk score 4_2 & 0.834 & $(0.75,0.93)$ & 0.002 & 0.976 & $(0.83,1.15)$ & 0.779 \\
\hline Behavioral risk score 4_3 & 0.867 & $(0.77,0.98)$ & 0.018 & 0.904 & $(0.75,1.09)$ & 0.283 \\
\hline Parent socioeconomic status & 0.960 & $(0.94,0.98)$ & 0.002 & 0.652 & $(0.49,0.86)$ & 0.003 \\
\hline Maternal obesity & 2.539 & $(2.17,2.97)$ & $<0.0001$ & 2.794 & $(2.08,3.75)$ & $<0.0001$ \\
\hline Genetic risk score & - & - & - & 1.092 & $(1.05,1.14)$ & $<0.0001$ \\
\hline
\end{tabular}

OR (Odds Ratio), $95 \% \mathrm{Cl}$ (95\% Confidence Interval)

*The analyses for the paired sample also controlled for genetic ancestry which is not displayed in the above table

can influence weight distribution and possibly have protective benefits against obesity in adulthood. To begin, breastfeeding appears to result in lower overall energy intake than formula feeding in the early postnatal period and is associated with an infant's ability to self-regulate milk intake [32, 33]. These findings take on greater significance when one considers the effect that early weight gain can have on weight status in later childhood and even adulthood. There is evidence that overweight status in childhood has an impact on the risk of being overweight in adulthood [33]. In addition, rapid weight gain in infancy is related to being overweight later in childhood [34].

A recent study that is most comparable to the current study was conducted by Rousseaux et al. [35]. The study used multivariate analysis to test for relationships with measures of body composition including WHtR. It found no significant associations between breastfeeding and measures of body composition. However, a nonsignificant trend towards a protective benefit was identified on the highest percentiles of abdominal adiposity. The two studies differed in the age of the participants in addition to a large difference in mean WHtR, which could account for the disparity in the findings. Given that HELENA is a cohort study, future research is warranted to test for the association in adulthood.
A previous study that is in conflict with the current study was a randomized control trial conducted in Belarus. This trial was a breastfeeding promotion intervention, and found that the intervention increased the duration of breastfeeding but did not influence weight or adiposity at roughly 6.5 years old [12]. However, the study was conducted in Belarus, a country that does not have a comparable prevalence of obesity to the United States. Therefore, the results may not be generalizable to the cohort used in the current study. In addition, this study influenced only duration of breastfeeding and not initiation. The current study compared individuals that were breastfed to those that were not breastfed. It may be possible that while long duration of breastfeeding doesn't add any additional risk reduction benefit, breastfeeding in early infancy may convey a significant benefit vs. individuals that were never breastfed at all.

Another study that contrasted with ours and used the Add Health sample was conducted by Evenhouse and Reilly. It found no differences in BMI between sibling pairs discordant for breastfeeding [15]. Utilizing a within-family discordant sibling model is an efficient approach to control for family-level effects including genetic ancestry, race, and SES. However, there are other differences between the two studies that could account for the contrasting findings. First, within-family analyses 
Table 6 Results of regression models predicting waist-to-height ratio, body mass index, and obesity from randomly selected subset of the sibling pair sample, $n=856$, Add Health Study 1994-2008

\begin{tabular}{|c|c|c|c|c|c|c|c|c|c|}
\hline & \multicolumn{3}{|c|}{ Waist-to-height ratio outcome* } & \multicolumn{3}{|c|}{ Body mass index outcome $e^{* *}$} & \multicolumn{3}{|c|}{ Body mass index $\geq 30$ outcome $e^{* * *}$} \\
\hline & $\bar{b}$ & SE & $P$ value & $\mathrm{b}$ & SE & $P$ value & $\overline{\mathrm{OR}}$ & $95 \% \mathrm{Cl}$ & $P$ value \\
\hline Intercept & 0.415 & 0.067 & $<0.0001$ & 19.782 & 5.060 & $<0.0001$ & 0 & 0 & 0 \\
\hline Sex & 0.042 & 0.007 & $<0.0001$ & 0.564 & 0.524 & 0.282 & 1.127 & $(0.83,1.53)$ & 0.439 \\
\hline Age & -0.001 & 0.002 & 0.665 & -0.045 & 0.147 & 0.762 & 0.993 & $(0.91,1.08)$ & 0.863 \\
\hline Breastfeeding & -0.025 & 0.008 & 0.001 & -1.604 & 0.576 & 0.005 & 0.695 & $(0.50,0.97)$ & 0.033 \\
\hline Birthweight $>2500 \mathrm{~g}$ & Reference & Reference & Reference & Reference & Reference & Reference & Reference & Reference & Reference \\
\hline Birthweight <2500 g & 0.006 & 0.010 & 0.524 & 0.174 & 0.749 & 0.816 & 1.213 & $(0.80,1.85)$ & 0.369 \\
\hline Birthweight Missing & 0.011 & 0.019 & 0.563 & 0.988 & 1.460 & 0.499 & 1.129 & $(0.49,2.58)$ & 0.774 \\
\hline Parent nonsmoker & Reference & Reference & Reference & Reference & Reference & Reference & Reference & Reference & Reference \\
\hline Parent smoker & 0.005 & 0.010 & 0.619 & 0.318 & 0.784 & 0.685 & 0.955 & $(0.61,1.50)$ & 0.840 \\
\hline Parent smoking missing & 0.005 & 0.010 & 0.625 & 0.724 & 0.726 & 0.319 & 0.998 & $(0.66,1.52)$ & 0.994 \\
\hline Non-Hispanic African American & Reference & Reference & Reference & Reference & Reference & Reference & Reference & Reference & Reference \\
\hline Non-Hispanic White & -0.022 & 0.030 & 0.455 & -2.317 & 2.228 & 0.299 & 0.382 & $(0.11,1.37)$ & 0.140 \\
\hline Non-Hispanic Asian/Native American & 0.022 & 0.053 & 0.682 & 2.978 & 3.954 & 0.452 & 0.378 & $(0.3,4.90)$ & 0.456 \\
\hline Hispanic & -0.034 & 0.028 & 0.227 & -2.587 & 2.138 & 0.227 & 0.594 & $(0.17,2.03)$ & 0.406 \\
\hline Behavioral risk score 4_1 & -0.013 & 0.004 & 0.001 & -0.534 & 0.305 & 0.080 & 0.827 & $(0.69,0.99)$ & 0.044 \\
\hline Behavioral risk score 4_2 & 0.000 & 0.004 & 0.990 & -0.044 & 0.328 & 0.894 & 1.038 & $(0.85,1.27)$ & 0.713 \\
\hline Behavioral risk score 4_3 & 0.003 & 0.006 & 0.616 & 0.296 & 0.419 & 0.480 & 1.174 & $(0.92,1.50)$ & 0.200 \\
\hline Parent socioeconomic status & -0.017 & 0.008 & 0.025 & -0.975 & 0.567 & 0.086 & 0.653 & $(0.47,0.91)$ & 0.011 \\
\hline Maternal obesity & 0.039 & 0.008 & $<0.0001$ & 3.339 & 0.619 & $<0.0001$ & 2.433 & $(1.72,3.45)$ & $<0.0001$ \\
\hline Genetic risk score & 0.005 & 0.001 & $<0.0001$ & 0.412 & 0.080 & $<0.0001$ & 1.079 & $(1.03,1.13)$ & 0.001 \\
\hline Ancestry principal component 1 & 0.575 & 0.599 & 0.338 & 36.065 & 45.094 & 0.424 & 0 & 0 & 0 \\
\hline Ancestry principal component 2 & -0.416 & 0.398 & 0.297 & -8.198 & 29.971 & 0.785 & 0 & 0 & 0 \\
\hline Ancestry principal component 3 & 0.982 & 0.237 & $<0.001$ & 67.069 & 17.867 & $<0.001$ & 0 & 0 & 0 \\
\hline Ancestry principal component 4 & 0.008 & 0.164 & 0.961 & 4.257 & 12.316 & 0.730 & 0 & 0 & 0 \\
\hline Ancestry principal component 5 & 0.346 & 0.205 & 0.092 & 29.949 & 15.419 & 0.052 & 0 & 0 & 0 \\
\hline
\end{tabular}

b (Coefficient) SE (Standard Error) OR (Odds Ratio) $95 \%$ Cl (95\% Confidence Interval)

* R-Squared $=0.18$

** R-Squared $=0.13$

*** This analysis also controlled for genetic ancestry which is not displayed in the above table

using discordant sibling pairs can be limited by withinfamily variation in breastfeeding. As noted in Evanhouse and Reilly, there were 523 sibling pairs discordant for breastfeeding duration and only 288 pairs discordant for breastfeeding (one sibling breastfed, the other did not). Given the relatively high correlation of BMI within sibling pairs (heritability estimates range from 60 to $80 \%$ ), it is possible that a discordant sibling pair study was underpowered to detect an association due to breastfeeding [36, 37]. Additionally, the present study used the participants BMI and WHtR at Wave 4 as opposed to Wave 1 , which would have reduced our sample size for pairs discordant for breastfeeding initiation to 62 and substantially reduced our statistical power to detect any differences between siblings discordant for breastfeeding. Finally, we observed that WHtR may be a more sensitive measure of body weight compared to $\mathrm{BMI}$ as it relates to the association with breastfeeding.

\section{Limitations}

One of the primary criticisms of breastfeeding studies in general is related to residual confounding and recall bias. In this study, we were not able to control for maternal diet, which is often implicated in early infant weight gain and childhood obesity. In addition, the information on breastfeeding duration was via self-report from the mothers. On average, mothers would have been asked about breastfeeding duration approximately $12-15$ years after the breastfeeding took place. It is unclear as to whether recall biases may exist in this regard. However, there aren't any reasons to expect systematic differences in recall for those individuals who have overweight children vs. those that do not. In addition, we analyzed 
breastfeeding strictly as an ever/never variable. While this has the detrimental effect of not discriminating between infants that were breastfed for a day and those that were breastfed for two years, it may have minimized some of the recall bias. Also, our control for maternal obesity was a self-reported measure at the time of the questionnaire. While this isn't an ideal measure of maternal obesity at the time of the child's birth, the rate of obesity in the UN sample (18.46\%) is consistent with NHANES measures from 1976 to 1980 (17.1 \%) [38], and we feel that the mother's weight status at the time of the parental survey may serve as an appropriate proxy for her status during and after pregnancy. Finally, we did not have information on whether or not the reported breastfeeding duration was exclusive or partial, and it is possible that the relationship between weight distribution and breastfeeding status could be affected by whether or not the breastfeeding was supplemented with solid food.

The genetic risk score consisted of 31 SNPs that had been validated using a sample of both black and white young adults from the Add Health Study [25]. As such, these SNPs were used to construct the genetic risk score in the current study. Genome-wide association studies have identified 90+ SNPs that are associated with body mass/obesity. However, for the purposes of this study, we were not focused on developing an optimal set of SNPs to predict body mass/obesity. Rather, our analysis was focused on an established set of SNPs that sufficiently accounts for the genetic risk of obesity [39-42]. While additional SNPs $(90+$ or including genome-wide SNPs) would result in a slightly better genetic risk score prediction, we are confident that adding the very small effects of these additional SNPs would not impact our primary analytic aim: relating breastfeeding to WHtR. Additionally, the measures of genetic ancestry were included to adjust our analyses for potential genetic (as opposed to racial/ethnic) differences on the genomewide scale.

Finally, the analysis was limited due to the availability of the genetic measures in the sibling pair sample. We tested for whether or not the relationship we observed between breastfeeding and measures of weight status in the sibling sample was an artifact of the relatedness of the participants. We randomly sampled one sibling from each pair and conducted the analyses on these participants. In all follow-up analyses, we observed the consistent, significant relationship between breastfeeding and measures of weight status. While other methods may be more efficient in the context of biologically related individuals (i.e., mixed effects regression) we were not interested in studying the family-level effects in these analyses. Furthermore, there are a limited number of sibling pairs discordant for breastfeeding, which diminishes our ability to assess any family-level impact on the association of interest.

\section{Conclusion}

Overall, we contend that our collective attention to race, SES, GRS, maternal obesity, and BRS would broadly serve as correlates of various lifestyle and body weight indicators. Residual confounding undoubtedly remains in these analyses. However, there would have to be substantial unmeasured confounding influence to be able to explain enough of the relationship between breastfeeding and weight distribution to completely ameliorate the associations observed in this study.

In conclusion, the results of the current study demonstrated that breastfeeding is inversely related to the WHtR in adulthood. While many studies have reported a relationship between breastfeeding duration and weight in childhood, few others have reported this association in a young adult population. Also the current study provided evidence that WHtR may be a more sensitive outcome variable than BMI for studies that investigate the association between breastfeeding and weight distribution or status. Finally, the current study demonstrated both the utility of using genetic factors underlying obesity as well as evaluating the robustness of the breastfeeding association controlling for these factors. We encourage future studies to investigate the mechanisms that may be responsible for the role of breastfeeding on weight distribution.

\section{Data availability statement}

All data used in this study is publicly available at the Add Health website: www.cpc.unc.edu/projects/addhealth.

\section{Supplementary information}

Supplementary information is available at BMC Public Health's website.

\section{Additional files}

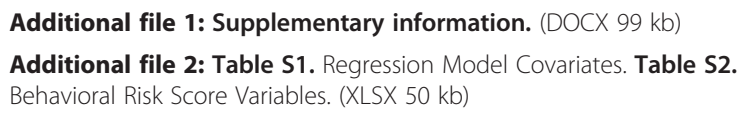

\section{Abbreviations}

BMI: body mass index; BRS: behavioral risk score; GRS: genetic risk score; PC: principal component; SES: socioeconomic status; SP: sibling pair sample; UN: unrelated sample; WHtR: waist-to-height ratio.

Competing interests

The authors of this paper have no competing interest.

Authors' contributions

$A B$ was the lead author on this paper. His contributions included study design, statistical analysis, and drafting of the manuscript. JB provided expertise on the social parameters measured as well as using the Add Health data set. BD provided coding and statistical expertise and constructed the 
BRS and GRS. MM was the graduate mentor of the lead author and provided guidance on the analysis and study design. All authors read and approved the final manuscript.

\section{Acknowledgements}

This research uses data from Add Health, a program project directed by Kathleen Mullan Harris and designed by J. Richard Udry, Peter S. Bearman, and Kathleen Mullan Harris at the University of North Carolina at Chapel Hill, and funded by grant P01-HD31921 from the Eunice Kennedy Shriver National Institute of Child Health and Human Development, with cooperative funding from 23 other federal agencies and foundations. Special acknowledgment is due Ronald R. Rindfuss and Barbara Entwisle for assistance in the original design. Information on how to obtain the Add Health data files is available on the Add Health website (http://www.cpc.unc.edu/addhealth). No direct support was received from grant P01-HD31921 for this analysis. Access and use of the Add Health data is supported by the University of Colorado Population Center (CUPC) which is funded in part by a grant from the Eunice Kennedy Shriver National Institute of Child Health and Human Development (NIH/NICHD R24HD066613) and the Population Program of the Institute of Behavioral Science.

\section{Author details}

'University of Colorado Boulder, 4185 47th St., Unit C, Boulder, CO 80301 USA. ${ }^{2}$ Institute of Behavioral Science, University of Colorado Boulder, 483 UCB, Boulder, CO 80309-0483, USA. ${ }^{3}$ Graduate School of Education, Stanford University, 502 Galvez Mall, Stanford, CA 94305, USA. Department of Integrative Physiology, University of Colorado Boulder, 354 UCB, Clare Small 102, Boulder, CO 80309-0354, USA

\section{Received: 12 February 2015 Accepted: 14 December 2015}

\section{Published online: 23 December 2015}

\section{References}

1. National Center for Health Statistics. National Health and Nutrition Examination Survey. Questionnaires, datasets, and related documentation. http://www.cdc.gov/nchs/ nhanes/nhanes_questionnaires.htm.

2. World Health Organization. Preventing chronic diseases: a vital investment. WHO Global Report: Geneva; 2005.

3. Binkley JK, Eales J, Jekanowski M. The relation between dietary change and rising US obesity. Int J Obes Relat Metab Disord. 2000;24(8):1032-9.

4. Nielsen SJ, Popkin BM. Patterns and trends in food portion sizes, 1977-1998. JAMA. 2003;289(4):450-3.

5. de Armas MG, Megias SM, Modino SC, Bolanos PI, Guardiola PD, Alvarez TM. Importance of breastfeeding in the prevalence of metabolic syndrome and degree of childhood obesity. Endocrinol Nutr. 2009;56(8):400-3.

6. Brion MJ, Lawlor DA, Matijasevich A, Horta B, Anselmi L, Araujo CL, et al. What are the causal effects of breastfeeding on IQ, obesity and blood pressure? evidence from comparing high-income with middle-income cohorts. Int J Epidemiol. 2011;40(3):670-80.

7. Gilman M, Rifas-Shiman SL, Camargo Jr CA, Berkey CS, Frazier AL, Rockett $\mathrm{HR}$, et al. Risk of overweight among adolescents who were breastfed as infants. JAMA. 2001;285(19):2461-7.

8. Hediger M, Overpeck MD, Kuczmarski RJ, Ruan WJ. Association between infant breastfeeding and overweight in young children. JAMA. 2001;284 2453-60.

9. Messiah SE, Arheart KL, Lipshultz SE, Bandstra ES, Miller TL. Perinatal factors associated with cardiovascular disease risk among preschool-age children in the united states: an analysis of 1999-2008 NHANES data. Int J Pediatr. 2012; 2012:157237.

10. Hunt KM, Foster JA, Forney LJ, Schutte UM, Beck DL, Abdo Z, et al. Characterization of the diversity and temporal stability of bacterial communities in human milk. PLoS One. 2011;6(6), e21313.

11. Saker M, Merzouk H, Merzouk SA, Ahmed SB, Narce M. Predictive factors of obesity and their relationships to dietary intake in schoolchildren in western algeria. Maedica (Buchar). 2011;6(2):90-9.

12. Kramer MS, Matush L, Vanilovich I, Platt RW, Bogdanovich N, Sevkovskaya Z, et al. Effects of prolonged and exclusive breastfeeding on child height, weight, adiposity, and blood pressure at age $6.5 \mathrm{y}$ : Evidence from a large randomized trial. Am J Clin Nutr. 2007;86(6):1717-21.

13. Parsons TJ, Power C, Manor O. Infant feeding and obesity through the lifecourse. Arch Dis Child. 2003;88(9):793-4.
14. Poulton R, Williams S. Breastfeeding and risk of overweight. JAMA. 2001; 286(12):1449-50.

15. Evenhouse E, Reilly S. Improved estimates of the benefits of breastfeeding using sibling comparisons to reduce selection bias. Health Ser Res. 2005; 40(6 Pt1):1781-802.

16. Nelson MC, Gordon-Larsen P, Adair LS. Are adolescents who were breastfed less likely to be overweight? Epidemiology. 2005;16(2):247-53.

17. Ashwell M, Gunn P, Gibson S. Waist-to-height ratio is a better screening tool than waist circumference and BMI for adult cardiometabolic risk factors: Systematic review and meta-analysis. Obes Rev. 2012;13(3):275-86.

18. Schneider HJ, Friedrich N, Klotsche J, Pieper L, Nauck M, John U, et al. The predictive value of different measures of obesity for incident cardiovascular events and mortality. J Clin Endocrinol Metab. 2010;95(4):1777-85.

19. Kuk JL, Katzmarzyk PT, Nichaman MZ, Church TS, Blair SN, Ross R. Visceral fat is an independent predictor of all-cause mortality in men. Obesity (Silver Spring). 2006;14:336-41.

20. Manolopoulos KN, Karpe F, Frayn KN. Gluteofemoral body fat as a determinant of metabolic health. Int J Obes (Lond). 2010;34:949-59.

21. Harris KM. The National Longitudinal Study of Adolescent Health (Add Health), Waves I \& II, 1994-1996; Wave III, 2001-2002; Wave IV, 2007-2009 [machine-readable data file and documentation]. Chapel Hill: Carolina Population Center, University of North Carolina at Chapel Hill; 2009. doi:10.3886/ICPSR27021.v9.

22. Stacklies W, Redestig H, Scholz M, Walther D, Selbig J. Pcamethods - a bioconductor package providing pca methods for incomplete data. Bioinformatics. 2007:23:1164-7.

23. Speliotes EK, Willer CJ, Berndt SI, Monda KL, Thorleifsson G, Jackson AU, et al. Association analyses of 249,796 individuals reveal 18 new loci associated with body mass index. Nat Genet. 2010;42(11):937-48. doi:10.1038/ng.686.

24. Monda KL, Chen GK, Taylor KC, Palmer C, Edwards TL, Lange LA, et al. A metaanalysis identifies new loci associated with body mass index in individuals of African ancestry. Nat Genet. 2013:45(6):690-6. doi:10.1038/ng.2608.

25. Domingue BW, Belsky DW, Mullan Harris KM, Smolen A, McQueen MB Boardman JD. Polygenic risk predicts obesity in both white and black young adults. PLoS One. 2014;9(7):e101596. doi:10.1371/journal.pone. 0101596.

26. Manichaikul A, Mychaleckyj JC, Rich SS, Daly K, Sale M, Chen WM. Robust relationship inference in genome-wide association studies. Bioinformatics. 2010;26(22):2867-73. doi:10.1093/bioinformatics/bta559.

27. McQueen MB, Boardman JD, Domingue BW, Smolen A, Tabor J, KilleyaJones L, et al. The National Longitudinal Study of Adolescent to Adult Health (Add Health) sibling pairs genome-wide data. Behav Genet. 2015; 45(1):12-23. doi:10.1007/s10519-014-9692-4.

28. Grummer-Strawn LM, Shealy KR. Progress in protecting, promoting, and supporting breastfeeding: 1984-2009. Breastfeed Med. 2009:4 Suppl 1:S31-9.

29. Belanoff CM, McManus BM, Carle AC, McCormick MC, Subramanian SV. Racial/ethnic variation in breastfeeding across the US: A multilevel analysis from the national survey of children's health, 2007. Matern Child Health J. 2012;16 Suppl 1:S14-26.

30. RStudio. RStudio: Integrated development environment for R (Version 0.97. 320) [Computer software]; 2012. Boston, MA. Retrieved Aug 9, 2012. Available from http://www.rstudio.org/.

31. Dietz WH. Health consequences of obesity in youth: childhood predictors of adult disease. Pediatrics. 1998;101(3 Pt 2):518-25.

32. Hester SN, Hustead DS, Mackey AD, Singhal A, Marriage BJ. Is the macronutrient intake of formula-fed infants greater than breast-fed infants in early infancy? J Nutr Metab. 2012;2012:891201.

33. Li R, Fein SB, Grummer-Strawn LM. Do infants fed from bottles lack selfregulation of milk intake compared with directly breastfed infants? Pediatrics. 2010:125(6):e1386-93.

34. Weng SF, Redsell SA, Swift JA, Yang M, Glazebrook CP. Systematic review and meta-analyses of risk factors for childhood overweight identifiable during infancy. Arch Dis Child. 2012;97(12):1019-26.

35. Rousseaux J, Duhamel A, Turck D, Molnar D, Salleron J, Artero EG, et al. Breastfeeding shows a protective trend toward adolescents with higher abdominal adiposity. Obes Facts. 2014;7(5):289-301.

36. Lee YS. The role of genes in the current obesity epidemic. Ann Acad Med Singapore. 2009;38(1):45-3.

37. Nan C, Guo B, Warner C, Fowler T, Barret T, Boomsma D, et al. Heritability of body mass index in pre-adolescence, young adulthood and late adulthood. Eur J Epidemiol. 2012;27(4):247-53. 
38. Baskin ML, Ard J, Franklin F, Allison DB. Prevalence of obesity in the united states. Obes Rev. 2005;6(1):5-7.

39. Hagg S, Ganna A, Van Der Laan SW, Esko T, Pers TH, Locke AE, et al. Genebased meta-analysis of genome-wide association studies implicates new loci involved in obesity. Hum Mol Genet. 2015;24(23):6849-60.

40. Cole CB, Nikpay M, Stewart AF, McPherson R. Increased genetic risk for obesity in premature coronary artery disease. Eur J Hum Genet. 2015. do:10. 1038/ejhg.2015.162.

41. Rukh G, Ahmed S, Ericson U, Hindy G, Stocks T, Renström F, et al. Inverse relationship between a genetic risk score of $31 \mathrm{BMI}$ loci and weight change before and after reaching middle age. Int J Obes. 2015. doi: 10.1038/ijo. 2015.180.

42. Todd JN, Dahlström EH, Salem RM, Sandholm N, Forsblom C, FinnDiane Study Group, et al. Genetic evidence for a causal role of obesity in diabetic kidney disease. Diabetes. 2015. [Epub ahead of print].

Submit your next manuscript to BioMed Central and we will help you at every step:

- We accept pre-submission inquiries

- Our selector tool helps you to find the most relevant journal

- We provide round the clock customer support

- Convenient online submission

- Thorough peer review

- Inclusion in PubMed and all major indexing services

- Maximum visibility for your research

Submit your manuscript at www.biomedcentral.com/submit
Biomed Central 